EPJ Web of Conferences 63,01011(2013)

DOI: 10.1051/epjconf/ 20136301011

(C) Owned by the authors, published by EDP Sciences, 2013

\title{
Level statistics of the spherical mean-field plus pairing model
}

\author{
Feng Pan ${ }^{1,2, a}$, Xin Guan ${ }^{1}$, Kristina D. Launey ${ }^{2}$, Jianzhong Gu$^{3}$, and Jerry P. Draayer ${ }^{2}$ \\ ${ }^{1}$ Department of Physics, Liaoning Normal University, Dalian 116029, China \\ ${ }^{2}$ Department of Physics and Astronomy, Louisiana State University, Baton Rouge, LA 70803-4001, USA \\ ${ }^{3}$ China Institute of Atomic Energy, P. O. Box 275(10), Beijing 102413, China
}

\begin{abstract}
The level statistics of the spherical mean-field plus pairing model is investigated based on the exact solutions obtained from the extended Heine-Stieltjes correspondence. It is shown that the level statistics for ${ }^{49} \mathrm{Ca}$ and ${ }^{50} \mathrm{Ca}$ calculated from the model with single-particle energies and a pairing strength extracted from experimental data indeed exhibits a chaotic behavior within the phase transitional region, while most isotopes display regular spectra.
\end{abstract}

\section{Introduction}

Random matrix theory (RMT) was first introduced in physics by Wigner [1] to study statistical properties of energy spectra of complex many-body quantum systems. There has been a considerable number of studies on the relation of quantum phase transitions and quantum chaos in finite quantum many-body systems. For example, Heiss et al. argued that the phase transitions in finite systems are likely to exhibit level statistics ascribed to quantum chaos [2]. They observed that a level repulsion in the spectrum occurs within the phase transitional region of a finite quantum system, and it is the level repulsion that is associated with the singular behavior of the spectrum, which occurs when the model parameter takes special values. These special (singular) points are called exceptional points. They conjectured that it is the distribution of the exceptional points that gives rise to the occurrence or absence of quantum chaos in the transitional region. In the interacting boson model (IBM), analysis with respect to the chaotic properties was performed $[3,4]$. It was observed that chaotic behaviors may occur in the vibrational to the rotational or the rotational to the $\gamma$-unstable shape phase transitional regions. Later on, these studies were extended to high-spin cases by including broken-pair degrees of freedom [5]. In some specific cases, there appears to be a definite relation between the singular behavior in the quantum spectrum and the singular behavior of the corresponding classical action of the associated Hamiltonian [6]. Level statistics of the single-particle levels generated from an axially symmetric potential with quadrupole and octupole deformations was also analyzed [7], which shows that chaotic motion does occur at the mean-field level for nuclei in such cases.

In this talk, we report our recent study on level statistics of a spherical mean-field plus pairing model. The model contains both a spherical mean field and the pairing interaction among valence nucleon pairs, which is exactly solvable. It has been found recently [8] that solutions of the model can be obtained from zeros of the associated extended Heine-Stieltjes polynomials, which makes it feasible to apply the model to many pairs over a large number of single-particle levels. Level statistical properties are studied for nuclear systems that are likely to be pairing-driven, namely, we investigate ${ }^{48-53} \mathrm{Ca}$ isotopes in the model with single-particle energies and a pairing strength deduced from experiment.

\section{The model and its exact solutions}

The Hamiltonian of the spherical mean-field plus pairing model within the proton or neutron sectors is given by

$$
\hat{H}=\sum_{j=1}^{n} \epsilon_{j} \hat{n}_{j}-G \sum_{j j^{\prime}} S_{j}^{+} S_{j^{\prime}}^{-},
$$

where the sums run over all given $j$-levels of total number $n, G>0$ is the overall pairing interaction strength, $\left\{\epsilon_{j}\right\}$ are nondegenerate single-particle energies obtained from any spherical mean-field theory, such as the spherical shell model, $\hat{n}_{j}=\sum_{m} a_{j m}^{\dagger} a_{j m}$ is the number operator for valence particles in the $j$-th level, and $S_{j}^{+}=\sum_{m>0}(-)^{j-m} a_{j m}^{\dagger} a_{j-m}^{\dagger}$ $\left(S_{j}^{-}=\left(S_{j}^{+}\right)^{\dagger}\right)$ are pair creation (annihilation) operators.

The exact $k$-pair eigenstates of (1) with $v_{j^{\prime}}=0$ for even systems or $v_{j^{\prime}}=1$ for odd systems, in which $j^{\prime}$ is the spin of the unpaired single particle, can be written as

$$
\left|k ; x ; v_{j^{\prime}}\right\rangle=S^{+}\left(x_{1}\right) S^{+}\left(x_{2}\right) \cdots S^{+}\left(x_{k}\right)\left|v_{j^{\prime}}\right\rangle,
$$

where $\left|v_{j^{\prime}}\right\rangle$ is the pairing vacuum state with the seniority $v_{j^{\prime}}$ that satisfies $S_{j}^{-}\left|v_{j^{\prime}}\right\rangle=0$ and $\hat{n}_{j}\left|v_{j^{\prime}}\right\rangle=\delta_{j j^{\prime}} v_{j}\left|v_{j^{\prime}}\right\rangle$ for all $j$, and

$$
S^{+}\left(x_{i}\right)=\sum_{j=1}^{n} \frac{1}{x_{i}-2 \epsilon_{j}} S_{j}^{+},
$$

\footnotetext{
ae-mail: daipan@dlut.edu.cn
} 
in which $x_{i}(i=1,2, \cdots, k)$ satisfy the following set of Bethe ansatz equations (BAEs):

$$
1-2 G \sum_{j} \frac{\rho_{j}}{x_{i}-2 \epsilon_{j}}-2 G \sum_{i^{\prime}=1(\neq i)}^{k} \frac{1}{x_{i}-x_{i^{\prime}}}=0,
$$

where the first sum runs over all $j$-levels and $\rho_{j}=-\Omega_{j} / 2$ with $\Omega_{j}=j+1 / 2-\delta_{j j^{\prime}} v_{j \prime}$. For each solution, the corresponding eigen-energy is given by

$$
E_{k}=\sum_{i=1}^{k} x_{i}+v_{j^{\prime}} \epsilon_{j^{\prime}}
$$

Through the Heine-Stieltjes correspondence, one can find the solutions [8] of the corresponding BAEs (4) by solving the second-order Fuchsian equation:

$$
A(x) y^{\prime \prime}(x)+B(x) y^{\prime}(x)-V(x) y(x)=0,
$$

where $A(x)=\prod_{j=1}^{n}\left(x-2 \epsilon_{j}\right)$ is a polynomial of degree $n$, the polynomial $B(x)$ is given as

$$
B(x) / A(x)=\sum_{j=1}^{n} \frac{2 \rho_{j}}{x-2 \epsilon_{j}}-\frac{1}{G},
$$

and $V(x)$ are called Van Vleck polynomials of degree $n-1$, which are determined according to Eq. (6). In search for polynomial solutions of Eq. (6), we write

$$
y(x)=\sum_{j=0}^{k} a_{j} x^{j}, \quad V(x)=\sum_{j=0}^{n-1} b_{j} x^{j}
$$

where $\left\{a_{j}\right\}$ and $\left\{b_{j}\right\}$ are the expansion coefficients to be determined. Substitution of (8) into Eq.(6) yields two matrix equations. By solving these two matrix equations, we can obtain [8] the solutions of $\left\{a_{j}\right\}$ and $\left\{b_{j}\right\}$.

Furthermore, if we set $a_{k}=1$ in $y(x)$, the coefficient $a_{k-1}$ becomes equal to the negative sum of the $y(x)$ zeros, $a_{k-1}=-\sum_{i=1}^{k} x_{i}$, and hence, yields the corresponding eigen-energy according to Eq. (5),

$$
E_{k}=-a_{k-1}+v_{j^{\prime}} \epsilon_{j^{\prime}}
$$

Thus, we can simultaneously obtain the energy spectrum consisting of all excited levels with angular momentum $J=0$ for even systems or $J=j^{\prime}$ for odd systems as well as the corresponding eigenstates given by Eq. (2).

\section{Numerical Results and Discussions}

We use two typical statistical measures to analyze the level fluctuation properties of a $k$-pair even or odd system. One is the nearest-neighbor level spacing distribution $P(S)$, and the other is the spectral rigidity $\Delta_{3}(L)$. These measures are determined through the unfolded levels $\left\{\tilde{E}_{k}(\mu)\right\}$ that are obtained from the mapping $N\left(E_{k}(\mu)\right) \mapsto \tilde{E}_{k}(\mu)$, where $\left\{E_{k}(\mu)\right\}$ are given by (5) with $\mu$ labeling the $\mu$-th excited level, and the mapping is determined by fitting a smooth polynomial function to the number staircase function, $N\left(E_{k}(\mu)\right)$, that counts the number of levels below
$E_{k}(\mu)$. By construction, the unfolded spectrum, $\left\{\tilde{E}_{k}(\mu)\right\}$, is dimensionless with an average level spacing of unity.

The distribution of the nearest-neighbor level spacing $P(S)$ is defined as the probability of two nearest-neighbor energy levels to have a spacing $S$, which is calculated from the unfolded spectrum as

$$
S_{\mu}=\tilde{E}_{k}(\mu+1)-\tilde{E}_{k}(\mu) .
$$

The distribution $P(S)$ is shown by normalized histograms in our analysis.

As is commonly accepted, Poisson statistics with

$$
P(S)=e^{-S},
$$

characterizes a regular system (uncorrelated level spacings), while the Wigner distribution, which is almost identical to the GOE prediction,

$$
P(S)=(\pi / 2) S e^{-\pi S^{2} / 4},
$$

characterizes a chaotic system (nearby levels likely to repel each other).

The spectral rigidity is defined as

$$
\Delta_{3}(\alpha, L)=\frac{1}{L} \min _{A, B} \int_{\alpha}^{\alpha+L}[N(\tilde{E})-(A \tilde{E}+B)]^{2} d \tilde{E},
$$

which is the average of the least-square deviations between the number staircase function $N(\tilde{E})$ for the unfolded spectrum and its best linear fit $(A \tilde{E}+B)$ over the energy interval $[\alpha, \alpha+L]$. For a given $L$, smaller values of $\Delta_{3}$ imply stronger long-term correlations between the levels.

The average of $\Delta_{3}(\alpha, L)$ over $n_{\alpha}$ intervals $(\alpha, \alpha+L)$, which overlap by $L / 2$ successively, yields a smoother $\bar{\Delta}_{3}(L)$ measure,

$$
\bar{\Delta}_{3}(L)=\frac{1}{n_{\alpha}} \sum_{\alpha} \Delta_{3}(\alpha, L) .
$$

It is shown that

$$
\bar{\Delta}_{3}(L)=L / 15
$$

for a regular spectrum, while, in the large- $L$ limit,

$$
\bar{\Delta}_{3}(L) \approx \frac{1}{\pi^{2}}(\ln L-0.0687),
$$

for a chaotic system with the GOE statistics.

We consider a realistic example with a focus on $\mathrm{Ca}$ isotopes that adopts single-particle energies as well as pairing strengths $G$ deduced from experimental data [9, 10]. We employ the model Hamiltonian (1) and study the ${ }^{48-53} \mathrm{Ca}$ level statistical properties associated with a dominant pairing two-body interaction.

The valence neutrons of the ${ }^{42-53} \mathrm{Ca}$ isotopes outside the ${ }^{40} \mathrm{Ca}$ core occupy five $j$-levels $1 f_{5 / 2,7 / 2}, 2 p_{1 / 2,3 / 2}$, and $1 g_{9 / 2}$ with $k=1,2, \ldots, 6$ pairs. For the odd- $A$ systems, the unpaired valance neutron is allowed to occupy the $f_{7 / 2}$ level for ${ }^{43-47} \mathrm{Ca}$ and the $p_{3 / 2}$ level for ${ }^{49-53} \mathrm{Ca}$, as indicated by the experimental ground-state spin-parity for these isotopes. The single-particle energies in this case are obtained from the ${ }^{40} \mathrm{Ca}$ and ${ }^{41} \mathrm{Ca}$ binding energies [9] and the excitation energies of ${ }^{41} \mathrm{Ca}$ [10], 


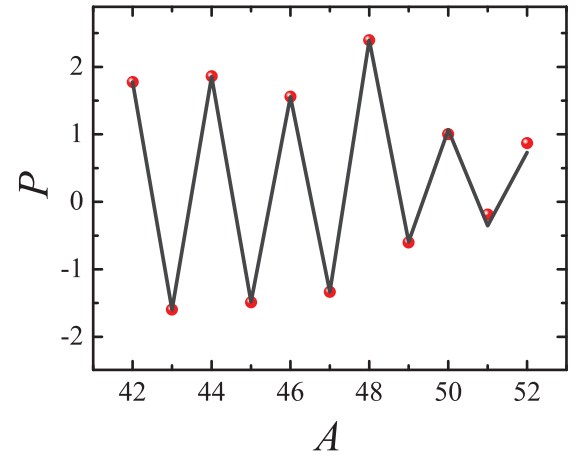

Figure 1. Theoretical values connected by solid lines and the corresponding experimental values (circles) deduced from Ref. [9] for the pairing gap $P$ (in units of $\mathrm{MeV}$ ) of ${ }^{42-52} \mathrm{Ca}$ as a function of the mass number $A$.

$$
\begin{aligned}
\epsilon_{7 / 2} & =-2.50 \mathrm{MeV}, \\
\epsilon_{3 / 2} & =-0.56 \mathrm{MeV}, \\
\epsilon_{5 / 2} & =0.08 \mathrm{MeV}, \\
\epsilon_{1 / 2} & =1.11 \mathrm{MeV}, \\
\epsilon_{9 / 2} & =1.95 \mathrm{MeV},
\end{aligned}
$$

which are used as an input to the model (1). We assume no proton excitations, which is a good approximation given the magic proton number for $\mathrm{Ca}$ isotopes, and hence, we can treat the energy due to protons as a constant.

The pairing interaction strength $G$ is determined from pairing gaps deduced from experimental binding energies [9]. The pairing gaps of ${ }^{42-53} \mathrm{Ca}$ are calculated by

$$
P(A)=E_{B}(A+1)+E_{B}(A-1)-2 E_{B}(A),
$$

where $E_{B}(A)$ is the binding energy of a nucleus with a mass number $A$. This formula is applied to experimental binding energies of $\mathrm{Ca}$ isotopes (Fig. 1, red circles), as well as to the ground-state energy of the pairing Hamiltonian (1), where the pairing strength $G$ used in (1) is determined in a way that the observed pairing gap is reproduced by the theoretical value for each $\mathrm{Ca}$ isotope in consideration (Fig. 1). The pairing strengths $G$ derived for ${ }^{42-53} \mathrm{Ca}$ are shown in Table 1.

Table 1. Pairing interaction strength $G$ deduced from experiment for the ${ }^{42-53} \mathrm{Ca}$ isotopes.

\begin{tabular}{cr|cr}
\hline Isotope & $G(\mathrm{MeV})$ & Isotope & $G(\mathrm{MeV})$ \\
\hline${ }^{42} \mathrm{Ca}$ & 0.4090 & ${ }^{43} \mathrm{Ca}$ & 0.4280 \\
${ }^{44} \mathrm{Ca}$ & 0.4214 & ${ }^{45} \mathrm{Ca}$ & 0.4440 \\
${ }^{46} \mathrm{Ca}$ & 0.4212 & ${ }^{47} \mathrm{Ca}$ & 0.4660 \\
${ }^{48} \mathrm{Ca}$ & 0.4385 & ${ }^{49} \mathrm{Ca}$ & 0.4400 \\
${ }^{50} \mathrm{Ca}$ & 0.3310 & ${ }^{51} \mathrm{Ca}$ & 0.2910 \\
${ }^{52} \mathrm{Ca}$ & 0.1750 & ${ }^{53} \mathrm{Ca}$ & 0.0300 \\
\hline
\end{tabular}

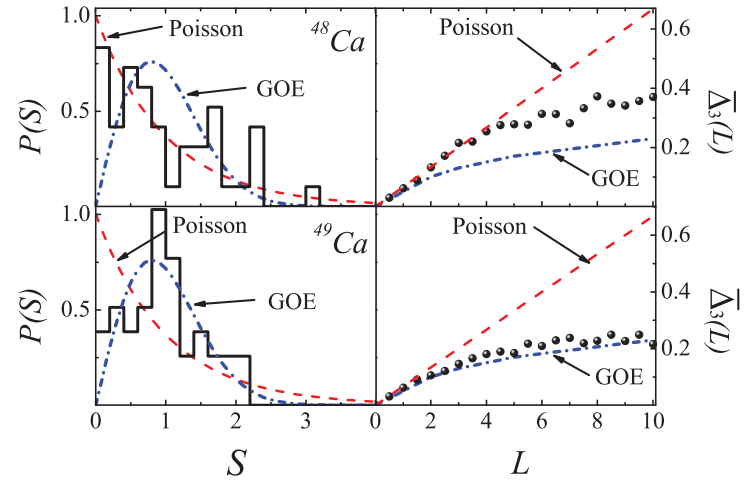

Figure 2. Level spacing distribution $P(S)$ (left panel) and spectral rigidity $\bar{\Delta}_{3}(L)$ (right panel) for the $J=0$ levels in ${ }^{48} \mathrm{Ca}$ with $G=0.4385 \mathrm{MeV}$ and for the $J=3 / 2$ levels in ${ }^{49} \mathrm{Ca}$ with $G=0.44 \mathrm{MeV} . P(S)$ and $\bar{\Delta}_{3}(L)$ for ${ }^{48} \mathrm{Ca}$ are of the Poisson type, while those for ${ }^{49} \mathrm{Ca}$ are of the GOE type.

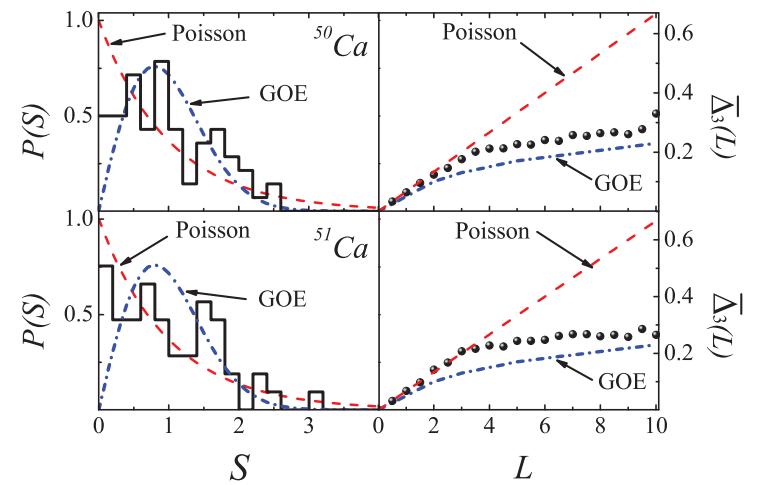

Figure 3. Level spacing distribution $P(S)$ (left panel) and spectral rigidity $\bar{\Delta}_{3}(L)$ (right panel) for the $J=0$ levels in ${ }^{50} \mathrm{Ca}$ with $G=0.331 \mathrm{MeV}$ and for the $J=3 / 2$ levels in ${ }^{51} \mathrm{Ca}$ with $G=0.291 \mathrm{MeV} . P(S)$ and $\bar{\Delta}_{3}(L)$ for ${ }^{50} \mathrm{Ca}$ are of the GOE type, while those for ${ }^{51} \mathrm{Ca}$ are of the Poisson type.

The spectral statistical properties for each $\mathrm{Ca}$ isotope are expected to be strongly influenced by the interplay of the mean-field and the pairing interaction. Namely, nuclear systems governed by small (but nonzero) or strong pairing are expected to obey Poisson statistics, while a GOE-type spectrum is likely to occur within the localized to superconducting transitional region. The level spacing distribution $P(S)$ and spectral rigidity $\bar{\Delta}_{3}(L)$ for ${ }^{48-53} \mathrm{Ca}$ are shown in Figs. 2-4. In most cases, such as ${ }^{48} \mathrm{Ca}$ and ${ }^{51-53} \mathrm{Ca}$, the $P(S)$ and $\bar{\Delta}_{3}(L)$ are of the Poisson type. The two exceptions, that are found to obey the GOE statistics, are ${ }^{49} \mathrm{Ca}$ and ${ }^{50} \mathrm{Ca}$, as revealed by the $P(S)$ and $\bar{\Delta}_{3}(L)$ quantities.

It is important to understand the origin of the GOE-like behavior for ${ }^{49} \mathrm{Ca}$ and ${ }^{50} \mathrm{Ca}$. In particular, as these isotopes are close to a subshell closure (neutron number 28) and 


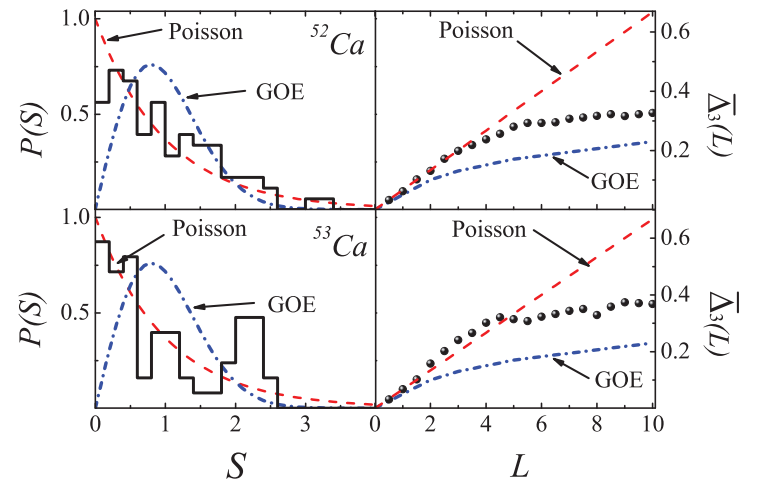

Figure 4. Level spacing distribution $P(S)$ (left panel) and spectral rigidity $\bar{\Delta}_{3}(L)$ (right panel) for the $J=0$ levels in ${ }^{52} \mathrm{Ca}$ with $G=0.175 \mathrm{MeV}$ and for the $J=3 / 2$ levels in ${ }^{53} \mathrm{Ca}$ with $G=0.03$ MeV. $P(S)$ and $\bar{\triangle}_{3}(L)$ for ${ }^{52} \mathrm{Ca}$ and ${ }^{53} \mathrm{Ca}$ are of the Poisson type.

hence, an effective ${ }^{48} \mathrm{Ca}$ core may be adopted, their unusual statistical behavior may be predominantly dictated by the shell structure. If this is the case, the spectrum would be effectively generated by one or two particles in a mean field, which would result in GOE-like statistical properties associated with the localized normal phase (e.g., the ground state of ${ }^{49} \mathrm{Ca}$ can be realized by the inactive 8 particles in the $1 f_{7 / 2}$ level and a particle in the $2 p_{3 / 2}$ ). However, the information entropy for the ground state of these nuclei is found to be much greater than zero. This points to the fact that, clearly, these systems are not in the localized phase and that there are important pairing correlations developed among all valence neutrons above a ${ }^{40} \mathrm{Ca}$ core. This, together with the GOE-like spectral statistics shown in Figs. 2 and 3, suggests that ${ }^{49} \mathrm{Ca}$ and ${ }^{50} \mathrm{Ca}$ realize a realistic example of nuclei in the phase transitional region from the localized to the superconducting phase. The GOE-like behavior of the level statistics at the exceptional $G$ points in the model may be due to the fact that these points are related locally to the behavior of the potential, representing generically the corresponding classical instability points, as conjectured in [7]. The results indicate that pairing-driven nuclei can indeed manifest a chaotic behavior, even though the regular behavior is more common.

\section{Conclusions}

In summary, we have studied the statistical properties of the energy spectra of the spherical mean-field plus pairing model. It is found that varying the pairing interac- tion strength $G$ in the model is likely to alter the statistical properties of the spectra. Specifically, the spectrum is regular when $G$ is either small but nonzero or $G$ is large enough. For these $G$ values, the system lies in the integrable normal or superconducting phases. There are many exceptional points of $G$ within the critical region from the normal (localized) to the nonlocal superconducting (pair condensate) phase transition in the model and at these points the level statistics is ascribed to quantum chaos. When the single-particle energies and pairing strength are deduced from the experiment, as shown by the statistical results for the levels in ${ }^{48-53} \mathrm{Ca}$ calculated from the model, we find that the exceptional points of $G$ indeed exist in realistic nuclear systems, even though spectra are more often regular.

Support from the U.S. National Science Foundation (OCI-0904874), U.S. Department of Energy (DESC0005248), the Southeastern Universities Research Association, the Natural Science Foundation of China (11175078, 10975190, and 11275271), the Doctoral Program Foundation of the State Education Ministry of China (20102136110002), and the LSU-LNNU joint research program (9961) is acknowledged.

\section{References}

[1] E. P. Wigner, Ann. Math. 53, 36 (1951); 62, 548 (1955); 65, 203 (1957); 67, 325 (1958).

[2] W. D. Heiss and A. L. Sannino, Phys. Rev. A 43, 4159 (1991).

[3] Y. Alhassid, A. Novoselsky, and N. Whelan, Phys. Rev. Lett. 65, 2971 (1990); Y. Alhassid, A. Novoselsky, Phys. Rev. C 45, 1677 (1992); Y. Alhassid and N. Whelan, Phys. Rev. Lett. 67, 816 (1991); Phys. Rev. C 43, 2637 (1991); N. Whelan and Y. Alhassid, Nucl. Phys. A 556, 42 (1993).

[4] P. Cejnar and J. Jolie, Phys. Rev. E 58, 387 (1998).

[5] Y. Alhassid and D. Vretenar, Phys. Rev. C 46, 1334 (1992).

[6] W. D. Heiss and M. Müller, Phys. Rev. E 66, 016217 (2002).

[7] W. D. Heiss, R. G. Nazmitdinov, and S. Radu, Phys. Rev. Lett. 72, 2351 (1994).

[8] X. Guan, K. D. Launey, M. Xie, L. Bao, F. Pan, and J. P. Draayer, Phys. Rev. C 86, 024313 (2012).

[9] G. Audi, A. H. Wapstra, and C. Thibault, Nucl. Phys. A 729, 337 (2003).

[10] J. A. Cameron and B. Singh, Nucl. Data Sheets 94, 429 (2001). 